\title{
STATIC SELF-GRAVITATING MANY-BODY SYSTEMS IN EINSTEIN GRAVITY
}

\author{
LARS ANDERSSON* AND BERND G. SCHMIDT
}

\section{Contents}

1. Introduction

2. General setup and solution of the projected system

3. Equilibration

4. Examples

References

\section{INTRODUCTION}

This paper continues the study of static self-gravitating elastic bodies in Einstein gravity which was initiated in [1]. In that paper, connected bodies were considered. Here, we shall consider the problem of constructing static, elastic, many-body systems in Einstein gravity.

In Newtonian gravity, one may easily construct static many-body systems consisting of rigid bodies. Newton showed that the potential inside a spherical, homogenous shell of matter is constant. It follows that a body placed at rest anywhere inside the shell, will remain at rest. This example generalizes to hollow ellipsoids, cf. [5].

The problem of constructing static, self-gravitating many-body configurations consisting of elastic bodies in Newtonian gravity was studied in [3]. One of the results proved there is that under certain conditions it is possible to place a small elastic body at a stationary point of the Newtonian potential of another body. We remark here that the above mentioned example of a body at rest inside a spherical shell is only possible in the case of elastic bodies if the smaller body is spherically symmetric and placed at the center of the spherical shell, see section 4.3 below.

The two main steps in the construction used in 11 are the application of the implicit function theorem to construct solutions of a certain projected version of the reduced, coupled system of Einstein and elastic equations, and an equilibration argument which showed that the solutions thus constructed are solutions of the full system. The implicit function theorem is used to deform from a relaxed body without gravity, i.e. with Newton's constant $G=0$, to a solution of the above mentioned projected, reduced system with $G \neq 0$. As was observed in $[2$ the linearization of the elastic system at a relaxed configuration has a cokernel corresponding precisely

Date: May 8, 2009.

* Supported in part by the NSF, under contracts no. DMS 0407732 and DMS-0707306. 
to the Killing fields of a Euclidean metric. It is in order to cancel this degeneracy that one is led to applying a projection to the system.

In generalizing the method used in the case of a connected body to the case of multiple bodies, it turns out that the linearized elastic system now has a cokernel corresponding to the product of the cokernels corresponding to each body. However, the equilibration argument developed in 1], which is essential to show that the solution constructed is a solution of the full coupled system of Einstein and elasticity equations, requires that one has a solution corresponding to a system which is projected along only one of the factors of this cokernel.

In order to achieve this situation one must in the case of multiple bodies, after solving the system resulting from projecting out the cokernel, perform a partial equilibration making use of the additional degrees of freedom one has in a multibody system provided by the relative position and orientation of the component bodies. This step requires certain non-degeneracy conditions on the Newtonian potential of the bodies, which are completely analogous to the ones considered in [3].

After the partial equilibration, the only remaining degeneracy corresponds to the cokernel associated with one of the component bodies, and the equilibration argument from [1 can be applied to finish the argument.

In sections 2 23, we present the field equations for self-gravitating elastic bodies and prove the existence of solutions to this system, for disconnected bodies, given certain non-degeneracy conditions on the relaxed reference system. In section 4 . we give a few concrete examples where this construction applies.

We remark that for simplicity, the results in sections 24 are stated in terms of self-gravitating two-body systems. However, the method and results apply with essentially no changes to the construction of $n$-body systems also for $n>2$. The point which needs to be noted here is that instead of partially equilibrating one of the bodies, cf. section 3. for the case of a system of $n$ bodies, it is necessary to partially equilibrate $n-1$ of the bodies, by a procedure completely analogous to the one presented here. We leave the details to the reader.

The construction of static solutions of the Einstein equations representing disconnected elastic bodies raises the question of the necessary conditions for the existence of such configurations. In particular, what condition rules out the existence of two static bodies in Einstein gravity?

In Newton's theory of gravity it may seem naively clear that two bodies can not remain at rest under their mutual gravitational force. However, it is important to note that in order to be able to make such a statement, one needs a separation condition. For example, consider a hollow sphere and place a small spherical body in the center. As is well known, the Newton potential is constant in the interior of a sphere, and therefore such a configuration is static.

A natural condition which rules out the existence of a static two-body system $\mathcal{B}_{1} \cup \mathcal{B}_{2}$ in Newtonian gravity is given by the existence of a plane which separates the two bodies $\mathcal{B}_{1}$ and $\mathcal{B}_{2}$. In this case it is easy to see that no static solution can exist. The self force of $\mathcal{B}_{1}$ vanishes. Consider now the component of the total force generated by $\mathcal{B}_{2}$ acting on $\mathcal{B}_{1}$, which is orthogonal to the separating plane. This component is clear non-vanishing and hence the bodies must begin to move.

Now consider the same question in Einstein's theory of gravity. As we show in this paper, there are static two-body configurations. However, in Einstein gravity, 
it is not clear how to generalize the separation condition discussed above from the Newtonian case. In the paper [4, it was proved that there are no static solutions of Einstein gravity which admit a separating, complete, totally geodesic hypersurface $\Sigma$. Here, separating can be taken to mean that $\Sigma$ lies in the complement of the bodies. In particular, this rules out a static two-body configuration where the bodies are separated by a totally geodesic hypersurface.

The problem under discussion, which is rather simple in Newton's theory, becomes a challenge in the context of Einstein's theory of gravity. One explanation for this additional difficulty is that in Einstein gravity, we loose the concept of force. Furthermore, the self field is no longer a useful concept because the field equations are nonlinear. Müller zum Hagen wrote a PhD thesis on this problem and has a result for two separated axially symmetric fluid bodies, cf. [7].

\section{General Setup And solution of the Projected System}

We adopt the notations and conventions of [1]. We here consider the situation where the reference body has two connected components,

$$
\mathcal{B}=\mathcal{B}_{1} \cup \mathcal{B}_{2} .
$$

The bodies are assumed to be disjoint domains in $\mathbb{R}_{\mathcal{B}}^{3}$, the extended body, and to have free boundaries. In particular, we consider, as in [1, the zero traction boundary conditions. We consider configurations $f: \mathbb{R}_{\mathcal{S}}^{3} \rightarrow \mathbb{R}_{\mathcal{B}}^{3}$ and deformations $\phi: \mathbb{R}_{\mathcal{B}}^{3} \rightarrow \mathbb{R}_{\mathcal{S}}^{3}$ as in the case of a connected body. Here $\mathbb{R}_{\mathcal{S}}^{3}$ is the space manifold. The same constitutive relations as in [1] are assumed to hold.

We will assume that $\mathcal{B}$ satisfies the Newtonian equilibrium condition

$$
\begin{aligned}
& \int_{\mathcal{B}_{\ell}} \xi^{i} \partial_{i} v=0, \quad \ell=1,2 \\
& \Delta v=4 \pi \stackrel{\circ}{ } \chi_{B}
\end{aligned}
$$

for any Killing field $\xi$ on $\mathbb{R}^{3}$, see the discussion in section 3.2 for explanation of this condition. Here $\stackrel{\AA}{\epsilon}$ is the rest mass term in the relativistic stored energy function, see [1, section 3.3] for discussion.

The field equations are the same as in the case of a connected body, namely in Lagrangian frame

$$
\begin{aligned}
\nabla_{j}\left(e^{U} \sigma_{i}{ }^{j}\right) & =e^{U}\left(n \epsilon-\sigma_{l}{ }^{l}\right) \nabla_{i} U \quad \text { in } f^{-1}(\mathcal{B}),\left.\quad \sigma_{i}{ }^{j} n_{j}\right|_{f^{-1}(\partial \mathcal{B})}=0 \\
\Delta_{h} U & =4 \pi G e^{U}\left(n \epsilon-\sigma_{l}{ }^{l}\right) \chi_{f^{-1}(\mathcal{B})} \quad \text { in } \mathbb{R}_{\mathcal{S}}^{3} \\
G_{i j} & =8 \pi G\left(\Theta_{i j}-e^{U} \sigma_{i j} \chi_{f^{-1}(\mathcal{B})}\right) \quad \text { in } \mathbb{R}_{\mathcal{S}}^{3}
\end{aligned}
$$

and in material frame

$$
\begin{aligned}
\nabla_{A}\left(e^{\bar{U}} \bar{\sigma}_{j}{ }^{A}\right) & =e^{\bar{U}}\left[\bar{\epsilon}-\frac{\bar{\sigma}_{l}^{l}}{\bar{n}}\right] \overline{\partial_{i} U} \quad \text { in } \mathcal{B},\left.\quad \bar{\sigma}_{i}{ }^{A} n_{A}\right|_{\partial \mathcal{B}}=0 \\
\overline{\Delta_{h} U} & =4 \pi G e^{\bar{U}}\left(\bar{n} \bar{\epsilon}-\bar{\sigma}_{l}^{l}\right) \chi_{\mathcal{B}} \quad \text { in } \mathbb{R}_{\mathcal{B}}^{3} \\
-\frac{1}{2} \overline{\Delta_{h} h_{i j}}+Q_{i j}(\bar{h}, \overline{\partial h}) & =2 \overline{\left(\nabla_{i} U\right)\left(\nabla_{j} U\right)}-8 \pi G e^{\bar{U}}\left(\bar{\sigma}_{i j}-\overline{h_{i j}} \bar{\sigma}_{l}^{l}\right) \chi_{\mathcal{B}} .
\end{aligned}
$$


2.1. Analytical setting. Let $B_{1}=W^{2, p}(\mathcal{B}) \times W_{\delta}^{2, p} \times E_{\delta}^{2, p}$, and let $B_{2}=\left[L^{p}(\mathcal{B}) \times\right.$ $\left.B^{1-1 / p, p}(\partial \mathcal{B})\right] \times L_{\delta-2}^{p} \times L_{\delta-2}^{p}$. Then, $B_{1}$ is a Banach manifold, and $B_{2}$ is a Banach space.

The residuals of equations (2.3) define a map $\mathcal{F}: \mathbb{R} \times B_{1} \rightarrow B_{2}, \mathcal{F}=\mathcal{F}(G, Z)$, where we use $Z=\left(\phi, \bar{U}, \overline{h_{i j}}\right)$ to denote a general element of $B_{1}$. We assume that $\phi$ is a diffeomorphism onto its image. Thus, $\mathcal{F}$ has components $\mathcal{F}=\left(\mathcal{F}_{\phi}, \mathcal{F}_{U}, \mathcal{F}_{h}\right)$, corresponding to the components of $B_{2}$, given by

$$
\begin{aligned}
& \mathcal{F}_{\phi}=\left(\nabla_{A}\left(e^{\bar{U}} \bar{\sigma}_{j}{ }^{A}\right)-e^{\bar{U}}\left[\bar{\epsilon}-\frac{\bar{\sigma}_{l}^{l}}{\bar{n}}\right] \overline{\partial_{i} U}, \quad \operatorname{tr}_{\partial \mathcal{B}}\left(\bar{\sigma}_{i}{ }^{A}\right) n_{A}\right) \\
& \mathcal{F}_{U}=\overline{\Delta_{h} U}-4 \pi G e^{\bar{U}}\left(\bar{n} \bar{\epsilon}-\bar{\sigma}_{l}^{l}\right) \chi_{\mathcal{B}} \\
& \mathcal{F}_{h}=-\frac{1}{2} \overline{\Delta_{h} h_{i j}}+Q_{i j}(\bar{h}, \overline{\partial h})-2 \overline{\nabla_{i} U \nabla_{j} U}+8 \pi G e^{\bar{U}}\left(\bar{\sigma}_{i j}-\overline{h_{i j}} \bar{\sigma}_{l}{ }^{l}\right) \chi_{\mathcal{B}}
\end{aligned}
$$

Recall, cf. [1, section 3.2], that in setting up the field equations in the material frame, an extension $\widehat{\phi}$ of $\phi$ from $\mathcal{B}$ to $\mathbb{R}_{\mathcal{B}}$ is used, cf. [1, Lemma 4.1]. The argument used to construct $\widehat{\phi}$ carries over without change to the case of a body with several connected components.

The equation to be solved is $\mathcal{F}(G, Z)=0$. The material form of the reference state is given by

$$
Z_{0}=\left(\mathbf{i}, 0, \hat{\delta}_{i j} \circ \mathbf{i}\right) \in B_{1} .
$$

The map $\mathcal{F}$ defined by (2.4) is easily verified to satisfy $\mathcal{F}\left(0, Z_{0}\right)=0$ and to map $B_{1} \rightarrow B_{2}$ locally near the reference state $Z_{0}$. As discussed in [1, the map $\mathcal{F}$ is smooth.

The Frechet derivative $D_{2} \mathcal{F}\left(0, Z_{0}\right)$ can be represented as the matrix of operators

$$
\left(\begin{array}{ccc}
D_{\phi} \mathcal{F}_{\phi} & D_{U} \mathcal{F}_{\phi} & D_{h} \mathcal{F}_{\phi} \\
0 & \Delta & 0 \\
0 & 0 & -\frac{1}{2} \Delta
\end{array}\right)
$$

(where the entries are evaluated at $\left(0, Z_{0}\right)$ ). In particular, the matrix is upper triangular, and the diagonal entries are isomorphisms, with the exception for $D_{\phi} \mathcal{F}_{\phi}\left(0, Z_{0}\right)$ which is Fredholm with nontrivial kernel and cokernel.

Let $\phi_{\ell}$ denote the restriction of $\phi$ to $\mathcal{B}_{\ell}$. Then we have in a natural way $\mathcal{F}=$ $\left(\mathcal{F}_{\phi_{1}}, \mathcal{F}_{\phi_{2}}, \mathcal{F}_{U}, \mathcal{F}_{h}\right)$, and $D_{\phi} \mathcal{F}_{\phi}\left(0, Z_{0}\right)$ takes the form

$$
\left(\begin{array}{cc}
D_{\phi_{1}} \mathcal{F}_{\phi_{1}} & 0 \\
0 & D_{\phi_{2}} \mathcal{F}_{\phi_{2}}
\end{array}\right)
$$

Let $\delta \bar{\sigma}_{i}{ }^{A}$ denote any combination of the Frechet derivatives of $\bar{\sigma}_{i}{ }^{A}$, evaluated at $\left(0, Z_{0}\right)$. Assuming we use a coordinate system $X^{A}$ where $V_{123}=1$, we have the relations

$$
0=\int_{\mathcal{B}_{\ell}} \xi^{i} \partial_{A}\left(\delta \bar{\sigma}_{i}{ }^{A}\right)-\int_{\partial \mathcal{B}_{\ell}} \xi^{i}\left(\delta \bar{\sigma}_{i}{ }^{A}\right) n_{A}, \quad \ell=1,2
$$

where $\mathcal{B}_{\ell}$ are the connected components of $\mathcal{B}$ and $n^{A}$ is the outward normal. This can be interpreted as saying that due to the natural boundary conditions, the linearized elasticity operator, restricted to each component of $\mathcal{B}$ is automatically equilibrated at the reference configuration $\left(0, Z_{0}\right)$. It follows that the cokernel of the operator

$$
D_{\phi} \mathcal{F}_{\phi}\left(0, Z_{0}\right): W^{2, p}(\mathcal{B}) \rightarrow\left[L^{p}(\mathcal{B}) \times B^{1-1 / p, p}(\partial \mathcal{B})\right]
$$


consists of the space

$$
\mathcal{Y}_{1} \oplus \mathcal{Y}_{2}
$$

where for $\ell=1,2, \mathcal{Y}_{\ell}$ is the space of Killing fields on $\mathcal{B}_{\ell}$ considered as a subset of $\left(\mathbb{R}_{\mathcal{B}}^{3}, \delta_{\mathcal{B}}\right)$, where $\delta_{\mathcal{B}}=\mathbf{i}^{*} \hat{\delta}$ is the Euclidean metric on $\mathbb{R}_{\mathcal{B}}^{3}$ induced from the Euclidean metric $\hat{\delta}$ on $\mathbb{R}_{\mathcal{S}}^{3}$. The fact that the range and cokernel of $\mathcal{F}_{\phi}$ consists of fields on $\mathcal{B}$ is due to the fact that we defined $\mathcal{F}$ by passing to the material frame.

Similarly, the kernel of $D_{\phi} \mathcal{F}_{\phi}\left(0, Z_{0}\right)$ is the space

$$
\mathcal{X}_{1} \oplus \mathcal{X}_{2}
$$

where $\mathcal{X}_{\ell}$ is the space of Killing fields on $\mathcal{B}_{\ell}$, considered as a subset of $\left(\mathbb{R}_{\mathcal{B}}^{3}, \delta_{\mathcal{B}}\right)$.

Hence, in view of ellipticity, the operator

$$
D_{\phi} \mathcal{F}_{\phi}\left(0, Z_{0}\right): W^{2, p}(\mathcal{B}) \rightarrow\left[L^{p}(\mathcal{B}) \times B^{1-1 / p, p}(\partial \mathcal{B})\right]
$$

is Fredholm with the finite dimensional kernel and cokernel discussed above.

2.2. Projections. Introduce the projection operators $\mathbb{P}_{\mathcal{B}_{\ell}}: B_{2} \rightarrow B_{2}$, which acts as the identity in the second and third components of $B_{2}$ and is defined in the first component of $B_{2}$ as the unique projection along the space of Killing fields on $\left(\mathcal{B}_{\ell}, \delta_{\mathcal{B}}\right)$, onto the range of $D_{\phi_{\ell}} \mathcal{F}_{\phi_{\ell}}\left(0, Z_{0}\right)$, which leaves the boundary data in the first component of $B_{2}$ unchanged. If we consider each component $\mathcal{B}_{\ell}$ separetely, the situation is analogous to the one for the case of a connected body, so that $\mathbb{P}_{\mathcal{B}_{\ell}} D_{\phi_{\ell}} \mathcal{F}_{\phi_{\ell}}\left(0, Z_{0}\right)$ is a surjection.

We now go back to letting the projection operator act on all components of $\mathcal{F}$, and define $\mathbb{P}_{\mathcal{B}_{1} \cup \mathcal{B}_{2}}=\mathbb{P}_{\mathcal{B}_{1}} \oplus \mathbb{P}_{\mathcal{B}_{2}}$ by combining the projections associated with each component of the body. Then we have that $\mathbb{P}_{\mathcal{B}_{1} \cup \mathcal{B}_{2}} D_{2} \mathcal{F}\left(0, Z_{0}\right)$ is a surjection.

The projected system has the property, as in the case of a connected body, cf. [1] that,

$$
\mathbb{P}_{\mathcal{B}_{1} \cup \mathcal{B}_{2}} D_{2} \mathcal{F}\left(0, Z_{0}\right)
$$

is a surjection with finite dimensional kernel. For this reason, the implicit function theorem can be applied more or less directly to construct solutions to the first system.

The data for an Euclidean motion is given by $A=\left(\alpha^{i}, \beta_{i j}\right)$, where $\alpha^{i} \in \mathbb{R}^{3}$ is a translation vector and $\beta_{i j}$ is an orthogonal matrix. The motion $A$ acts in $\mathbb{R}_{\mathcal{S}}^{3}$ by $x \mapsto \beta(x+\alpha)$. Denote the group of Euclidean motions by $\mathcal{A}$, and let $I \in \mathcal{A}$ be the identity. We shall consider situations where to leading order $\phi$ maps $\mathcal{B}_{2}$ to $A \mathbf{i}\left(\mathcal{B}_{2}\right)$. We implement this by putting conditions on the the 1-jet of $\phi$ at a point $X_{2} \in \mathcal{B}_{2}$.

2.3. Solving the projected equation. The following result is analogous to [1, Proposition 4.3]. However, here we construct a family of solutions to the first projected system with parameters $(G, A)$, where $A \in \mathcal{A}$. The proof is an application of the implicit function theorem.

Proposition 2.1. Let $\mathcal{F}: B_{1} \rightarrow B_{2}$ be map defined by 2.4 and let $\mathbb{P}_{\mathcal{B}_{1} \cup \mathcal{B}_{2}}$ be defined as in section 2.2. Let $X_{1} \in \mathcal{B}_{1}, X_{2} \in \mathcal{B}_{2}$ be given points, and let $A$ be an Euclidean motion. Then, for sufficiently small values of Newton's constant $G$, and for $A$ sufficiently close to $I$, there is a solution $Z=Z(G, A)$, where $Z=\left(\phi, \bar{U}, \overline{h_{i j}}\right)$, to the reduced, projected equation for self-gravitating elastostatics given by

$$
\mathbb{P}_{\mathcal{B}_{1} \cup \mathcal{B}_{2}} \mathcal{F}(G, Z)=0,
$$


satisfying the conditions

$$
\begin{aligned}
(\phi-\mathbf{i})^{i}\left(X_{1}\right) & =0, \quad \delta^{C}{ }_{i} \delta_{C[A} \partial_{B]}(\phi-\mathbf{i})^{i}\left(X_{1}\right)=0 \\
(\phi-A \circ \mathbf{i})^{i}\left(X_{2}\right) & =\alpha^{i}, \quad \delta^{C}{ }_{i} \delta_{C[A} \partial_{B]}(\phi-A \circ \mathbf{i})^{i}\left(X_{2}\right)=\beta_{A B}
\end{aligned}
$$

In particular, for any $\epsilon>0$, there is a $G>0$, such that $Z=Z(G, A)$ satisfies the inequality

$$
\|\phi-\mathbf{i}\|_{W^{2, p}\left(\mathcal{B}_{1}\right)}+\|\phi-A \circ \mathbf{i}\|_{W^{2, p}\left(\mathcal{B}_{2}\right)}+\left\|\overline{h_{i j}}-\delta_{i j}\right\|_{W_{\delta}^{2, p}}+\|\bar{U}\|_{W_{\delta}^{2, p}}<\epsilon .
$$

\section{Equilibration}

In this section we will make use of the solution to the projected system (2.5) to construct solutions of the full system of Einstein equations for two static elastic bodies. Given a solution to the projected system (2.5) as in Proposition 2.1. our first goal is to construct a family of solutions to (2.5) which are equilibrated on one component of the body. Once this is done, we are in a situation where we are able to apply the equilibration argument presented in [1, section 5] to construct solutions to the full system of equations for the self-gravitating elastic body with two components.

3.1. Partial Equilibration. For definiteness we shall focus on $\mathcal{B}_{2}$ and construct a curve $Z=Z(G)$ such that

$$
\int_{\mathcal{B}_{2}} \xi^{i} b_{i}=0
$$

for all Killing fields $\xi^{i}$ on $\mathbb{R}_{\mathcal{B}}^{3}$, where

$$
b_{i}=\nabla_{A}\left(e^{\bar{U}} \bar{\sigma}_{j}^{A}\right)-e^{\bar{U}}\left[\bar{\epsilon}-\frac{\bar{\sigma}_{l}^{l}}{\bar{n}}\right] \overline{\partial_{i} U}
$$

is the first component of $\mathcal{F}_{\phi}$. We do this by finding $A=A(G)$, with $A(0)=I$, such that $Z(G, A(G))$ solves (3.1) as an equation for $A=A(G)$.

3.2. The normalized force. Let $\left(\xi_{(\alpha)}^{i}\right)_{\alpha=1}^{6}$ be a basis for the space of Killing fields on $\mathbb{R}_{\mathcal{B}}^{3}$. It will be convenient to solve (3.1) by transforming to the Eulerian frame, using the change of variables formula as in [1, section 5.1].

We define the force map $\widetilde{\mathbb{N}}=\left(\widetilde{\mathbb{N}}_{(\alpha)}(G, Z(G, A))\right)_{\alpha=1}^{6}, \widetilde{\mathbb{N}}: \mathbb{R} \times \mathcal{A} \rightarrow \mathbb{R}^{6}$, by

$$
\widetilde{\mathbb{N}}_{(\alpha)}(G, A)=\int_{\phi\left(\mathcal{B}_{2}\right)} \xi_{(\alpha)}^{i} \circ \phi^{-1}\left[\nabla_{j}\left(e^{U} \sigma_{i}{ }^{j}\right)-e^{U}\left(n \epsilon-\sigma_{l}{ }^{l}\right) \nabla_{i} U\right] d \mu_{h}
$$

where the right hand side is evaluated at $Z(G, A)$. The form of the force map in the material frame is easily found by analogy with (3.1). We write this as

$$
\widetilde{\mathbb{N}}_{(\alpha)}=\int_{\mathcal{B}_{2}} \xi_{(\alpha)}^{i} b_{i}
$$

We will freely make use of the form of $\widetilde{\mathbb{N}}$ which is most convenient. Since there is a factor $G$ in equation $2.2 \mathrm{~b}$ we see that $\widetilde{\mathbb{N}}(0, A)=0$. Hence it is convenient to introduce a normalied force map $\mathbb{N}$ for $G \neq 0$ by setting

$$
\mathbb{N}=G^{-1} \widetilde{\mathbb{N}}
$$

We define $\mathbb{N}(0, A)$ by taking the limit as $G \rightarrow 0$, which is easily shown to be well defined, see below. It is natural to view $\widetilde{\mathbb{N}}$ and $\mathbb{N}$ as taking values in the dual of 
$\operatorname{Lie}(\mathcal{A}) \cong \mathbb{R}^{6}$. If we denote by $\langle\cdot, \cdot\rangle$ the pairing between $\operatorname{Lie}(\mathcal{A})$ and its dual, then we can write eg.

$$
\langle\mathbb{N}, \xi\rangle=G^{-1} \int_{\mathcal{B}_{2}} \xi^{i} b_{i}
$$

Introducing a new potential $V$ by $G V=U$, we have

$$
\mathbb{N}_{(\alpha)}=\int_{\phi\left(\mathcal{B}_{2}\right)} \xi_{(\alpha)}^{i} \circ \phi^{-1}\left[\nabla_{j}\left(e^{G V} G^{-1} \sigma_{i}{ }^{j}\right)-e^{G V}\left(n \epsilon-\sigma_{l}{ }^{l}\right) \nabla_{i} V\right] d \mu_{h}
$$

where from (2.2b), $V$ and $h$ solve

$$
\begin{aligned}
\Delta_{h} V & =4 \pi e^{G V}\left(n \epsilon-\sigma_{l}^{l}\right) \chi_{\phi(\mathcal{B})} \quad \text { in } \mathbb{R}_{\mathcal{S}}^{3}, \\
G_{i j} & =8 \pi G\left(G^{2} \Theta[V]_{i j}-e^{G V} \sigma_{i j} \chi_{f^{-1}(\mathcal{B})}\right) \text { in } \mathbb{R}_{\mathcal{S}}^{3}
\end{aligned}
$$

3.3. Newtonian Equilibrium condition. In order to evaluate $\mathbb{N}$ at $(0, I)$ we

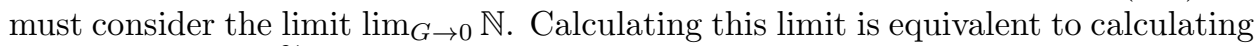
the derivative $\partial_{G} \widetilde{\mathbb{N}}(0, I)$. Differentiating the system (3.4) with respect to $G$, at $G=0$, we find

$$
\begin{aligned}
\mathbb{N}_{(\alpha)}(0, A) & \left.=\int_{A \mathbf{i}\left(\mathcal{B}_{2}\right)} \xi^{i}\left[\partial_{j}(\delta \sigma)_{i}{ }^{j}\right)-\stackrel{\circ}{\epsilon} \partial_{i} V\right], \\
\Delta V & =4 \pi €\left(\chi_{\mathbf{i}\left(\mathcal{B}_{1}\right)}+\chi_{A \mathbf{i}\left(\mathcal{B}_{2}\right)}\right)
\end{aligned}
$$

where $\delta \sigma_{i}{ }^{j}$ is a collection of derivatives of $\sigma_{i}{ }^{j}$ with respect to $\phi$. The first term in the right hand side of (3.5a) vanishes identically, cf. the discussion in section 2.1. see also [1, §4.2]. Therefore we have

$$
\begin{aligned}
\mathbb{N}_{(\alpha)}(0, A) & =\int_{A \mathbf{i}\left(\mathcal{B}_{2}\right)} \xi_{(\alpha)}^{i} \partial_{i} V, \\
\Delta V & =4 \pi \epsilon\left(\chi_{\mathbf{i}\left(\mathcal{B}_{1}\right)}+\chi_{A \mathbf{i}\left(\mathcal{B}_{2}\right)}\right)
\end{aligned}
$$

for Killing fields $\xi_{(\alpha)}$. We shall look for a family of solutions of $\mathbb{N}(G, A)=0$ of the form $A=A(G)$, with $A(0)=I$. For this to be possible, it is necessary that the condition $\mathbb{N}(0, I)=0$ holds. Let $V_{\ell}$ be the Newtonian potential of the components $\mathcal{B}_{\ell}$. In view of the third axiom of Newton, the principle of actio est reactio, cf. [3, $\S 5]$, the self-force of a body vanishes. Applying this to each component and to the whole body we have

$$
0=\int_{\mathcal{B}_{\ell}} \xi^{i} \partial_{i} V_{\ell}, \quad \ell=1,2,
$$

and

$$
0=\int_{\mathcal{B}_{1}} \xi^{i} \partial_{i} V_{2}+\int_{\mathcal{B}_{2}} \xi^{i} V_{1}
$$

This implies that $\mathbb{N}(0, I)=0$ takes the form

$$
0=\int_{\mathcal{B}_{2}} \xi^{i} \partial_{i} V_{1}
$$

for Killing fields $\xi^{i}$. We are assuming that (3.8) holds for the reference configuration, cf. (2.1a). In particular we have

$$
\begin{aligned}
\mathbb{N}_{(\alpha)}(0, A) & =\int_{A \mathbf{i}\left(\mathcal{B}_{2}\right)} \xi_{(\alpha)}^{i} \partial_{i} V_{1}, \\
\Delta V_{1} & =4 \pi \hat{\epsilon} \chi_{\mathbf{i}\left(\mathcal{B}_{1}\right)}
\end{aligned}
$$


for Killing fields $\xi_{(\alpha)}$.

3.4. Effect of motions on the normalized force. Next we consider the derivative $\partial_{A} \mathbb{N}(0, I)$. We must consider the $A$ derivative of equation (3.9a) at $G=0$. To do this, we must consider the effect on $A \mathbf{i}\left(\mathcal{B}_{2}\right)$ of its motion in the potential of $\mathbf{i}\left(\mathcal{B}_{1}\right)$. Let $A=I+\epsilon \eta+O\left(\epsilon^{2}\right)$, where $\eta$ is the infinitesimal motion with data $(\alpha, \beta)$, i.e. the Killing field $\eta^{i}=\alpha^{i}+\beta^{i}{ }_{j} x^{j}$. Then we have $A^{-1}=I-\epsilon \eta+O\left(\epsilon^{2}\right)$, so that

$$
\left.\partial_{A}\left(A^{-1}\right)\right|_{A=I} \cdot \eta=-\eta
$$

By the change of variables formula

$$
\int_{A \mathbf{i}\left(\mathcal{B}_{2}\right)} \xi_{(\alpha)}^{i} \partial_{i} V_{1}=\int_{\mathbf{i}\left(\mathcal{B}_{2}\right)}\left(\xi_{(\alpha)}^{i} \partial_{i} V_{1}\right) \circ A^{-1}
$$

Differentiating the integral with respect to $A$ at $I$ in the direction $\eta$ gives

$$
\int_{\mathbf{i}\left(\mathcal{B}_{2}\right)}\left[\xi_{(\alpha)}, \eta\right]^{i} \partial_{i} V_{1}-\xi_{(\alpha)}^{i} \partial_{i} \partial_{m} V_{1} \eta^{m}
$$

The Lie bracket $\left[\xi_{(\alpha)}, \eta\right]$ is again a Killing field, and hence in view of the fact that, by assumption, each component is equilibrated separately, cf. equation (3.8), the first term integrates to zero. This leads to

$$
\partial_{A} \mathbb{N}_{(\alpha)}(0, I) \cdot \eta=-\int_{\mathbf{i}\left(\mathcal{B}_{2}\right)} \xi_{(\alpha)}^{i} \partial_{i} \partial_{m} V_{1} \eta^{m}
$$

We may view $\partial_{A} \mathbb{N}(0, I)$ as a linear map $\mathbb{R}^{6} \rightarrow \mathbb{R}^{6}$. If this is invertible, we may again apply the implicit function theorem and solve $\mathbb{N}=0$.

Proposition 3.1. Assume that the reference body $\mathcal{B} \subset \mathbb{R}_{\mathcal{B}}^{3}$ is in equilibrium in the sense that (2.1a) holds. Let $Z=Z(G, A)$ be the solution to $\mathbb{P}_{\mathcal{B}_{1} \cup \mathcal{B}_{2}} \mathcal{F}=0$ constructed in Proposition [2.1, and let $\mathbb{N}(G, A)$ be the normalized force map defined by (3.3).

Suppose that the derivative $\partial_{A} \mathbb{N}(0, I): \mathbb{R}^{6} \rightarrow \mathbb{R}^{6}$ is invertible. Then there is an $\epsilon>0$ and a smooth map $G \mapsto A(G),[0, \epsilon) \rightarrow \mathcal{A}$ with

$$
\mathbb{N}(G, A(G))=0
$$

for $G \in[0, \epsilon)$.

3.5. Equilibration. We are now in a position to apply the method developed in [1] to complete the construction of solutions to the full system (2.3), which then also gives a solution to (2.2). If the assumptions of Proposition 3.1 hold, then we may assume without loss of generality that $\mathcal{B}_{2}$ is equilibrated. Therefore we are in a situation which is completely analogous to that considered in [1, §5], and a straightforward application of the methods developed there yields the following result.

Theorem 3.2. Let $Z(G, A)$ be the solution to the reduced, projected system of equations for a static, elastic, self-gravitating body

$$
\mathbb{P}_{\mathcal{B}_{1} \cup \mathcal{B}_{2}} \mathcal{F}=0
$$

constructed in Proposition 2.1. Assume that the normalized force map satisfies

$$
\mathbb{N}(G, A(G))=0 \text {. }
$$

Then in fact $Z(G, A(G))$ solves the full system (2.2) of equations for a static, elastic, self-gravitating body. 
The following is an immediate corollary of proposition 3.1 and theorem 3.2 .

Corollary 3.3. Assume that the reference body $\mathcal{B} \subset \mathbb{R}_{\mathcal{B}}^{3}$ is in equilibrium in the sense that 2.1a) holds. Let $Z=Z(G, A)$ be the solution to $\mathbb{P}_{\mathcal{B}_{1} \cup \mathcal{B}_{2}} \mathcal{F}=0$ constructed in Proposition 2.1, and let $\mathbb{N}(G, A)$ be the normalized force map defined by (3.3).

Suppose that the derivative $\partial_{A} \mathbb{N}(0, I): \mathbb{R}^{6} \rightarrow \mathbb{R}^{6}$ is invertible. Then there is an $\epsilon>0$ and a smooth map $G \mapsto A(G),[0, \epsilon) \rightarrow \mathcal{A}$ such that $Z=Z(G, A(G))$ is a solution to the full system (2.2) of equations for a static, elastic, self-gravitating body.

We have now reduced the problem of construcing a static self-gravitating twobody system to the question of whether the normalized force map has the property that $\partial_{A} \mathbb{N}(0, I)$ is invertible. This is clearly determined by the properties of the reference body $\mathcal{B}$. In section 4 below, we consider some particular cases.

\section{EXAMPLES}

In this section we give some examples of situations where the results developed in this paper apply. In view of corollary 3.3 , the it suffices to that normalized force map of the reference body has invertible Jacobian. This condition on the reference body is precisely equivalent to the condition needed for the case of static elastic Newtonian two-body systems considered in [3, section 5]. In general, for each example considered there, we have a corresponding example of a an static elastic self-gravitating two-body system in Einstein gravity. We shall here present an independent analysis of these constructions.

4.1. Small body. Here we consider a situation analogous to the one discussed in [3. section 5]. Let $\mathcal{B}_{1}$ be given and let $V_{1}$ be the Newtonian potential of $\mathbf{i}\left(\mathcal{B}_{1}\right)$. Assume $V_{1}$ has a non-degenerate stationary point, which we may without loss of generality assume to be at the origin $\mathcal{O}$ of the cartesian coordinate systems on $\mathbb{R}_{\mathcal{B}}^{3}$ and $\mathbb{R}_{\mathcal{S}}^{3}$. Thus, $\partial_{i} V_{1}$ is of the form

$$
\partial_{i} V_{1}=B_{i j} x^{j}+O\left(|x|^{2}\right)
$$

We may without loss of generality, after rotating the coordinate system, assume that $B_{i j}$ is diagonal, $B_{i j}=\sigma_{i} \delta_{i j}$.

We consider a test body $\mathcal{B}_{2}$. We may without loss of generality assume that $\mathcal{B}_{2}$ has its center of mass at the origin $\mathcal{O}$, i.e.

$$
\int_{\mathbf{i}\left(\mathcal{B}_{2}\right)} x^{i}=0, \quad i=1,2,3
$$

Define

$$
J^{i j}=\int_{\mathcal{B}_{2}} x^{i} x^{j}
$$

We call $J^{i j}$ as the tensor of inertia of $\mathcal{B}_{2}$, it should however be noted that the standard usage, cf. [6, section 5.3] is to define the inertia tensor as

$$
\int_{\mathcal{B}_{2}}|x|^{2} \delta^{i j}-x^{i} x^{j}
$$


We will now show that there is a homothety $F$ of $\mathbb{R}_{\mathcal{S}}^{3}$ such that $F \mathbf{i}\left(\mathcal{B}_{2}\right)$ is equilibrated with respect to the Newtonian potential $V_{1}$, i.e.

$$
\int_{F \mathbf{i}\left(\mathcal{B}_{2}\right)} \xi^{i} \partial_{i} V_{1}=0, \quad \forall \text { Killing fields } \xi \text { of } \mathbb{R}_{\mathcal{S}}^{3} .
$$

A homothetic motion of $\mathbb{R}_{\mathcal{S}}^{3}$ can be written in the form

$$
F x=\lambda Q(x+p)
$$

where $\lambda>0$ is a scale factor, $Q$ is a rotation and $p \in \mathbb{R}^{3}$ is a translation. We first consider homotheties of the form $F(\lambda, p) x=\lambda(x+p)$. Then by the change of variables formula and (4.1), we have for $\xi^{i}=\alpha^{i}$,

$$
\int_{F \mathbf{i}\left(\mathcal{B}_{2}\right)} \xi^{i} \partial_{i} V^{i}=\lambda^{4} \int_{\mathbf{i}\left(\mathcal{B}_{2}\right)} \alpha^{i} B_{i j}\left(x^{j}+p^{j}\right)+O\left(\lambda^{5}\right)
$$

Thus, defining the normalized force map $\mathbb{R}_{+} \times \mathbb{R}^{3} \rightarrow \mathbb{R}^{3}$ by

$$
\mathbb{N}_{i}(\lambda, p)=\lambda^{-4} \int_{F(\lambda, p) \mathbf{i}\left(\mathcal{B}_{2}\right)} \partial_{i} V
$$

we have $\mathbb{N}(0,0)=0$ and

$$
\partial_{p^{j}} \mathbb{N}_{i}(0,0)=\left|\mathcal{B}_{2}\right| B_{i j}
$$

Thus, if the matrix $B_{i j}$ is invertible, then we may apply the implicit function theorem to conclude that for small $\lambda>0$, there is a smooth function $p(\lambda)$ satisfying $p(0)=0$ such that with $F(\lambda, p(\lambda)) \mathbf{i}(\mathcal{B}))$ is equilibrated. In the following we will consider this case only.

Next, we consider homotheties of the form $F(\lambda, Q)=\lambda(Q x+p(\lambda))$. After applying the transformation $x \rightarrow \lambda(x+p(\lambda)), \mathcal{B}_{2}$ is equilibrated with respect to translational Killing fields. Thus it is sufficient to consider rotational Killing fields $\xi^{i}(x)=\beta^{i}{ }_{j} x^{j}$ and motions of the form

$$
F(\lambda, Q)=\lambda(Q x+p(\lambda))
$$

The change of variables formula and (4.1) gives, after taking into account the fact that $p(\lambda)=O(\lambda)$,

$$
\int_{F \mathbf{i}\left(\mathcal{B}_{2}\right)} \xi^{i} \partial_{i} V=\lambda^{5} \int \beta_{n}^{i} Q^{n} j x^{j} B_{i m} Q^{m}{ }_{k} x^{k}+O\left(\lambda^{6}\right)
$$

Let $\mathrm{SO}(3)$ be the group of rotations of $\mathbb{R}_{\mathcal{S}}^{3}$, and consider the normalized torque map

$$
\mathbb{T}: \mathbb{R}_{+} \times \mathrm{SO}(3) \rightarrow \mathbb{R}^{3}
$$

defined by

$$
\mathbb{T}_{(\alpha)}(\lambda, Q)=\lambda^{-5} \int_{\lambda Q \mathbf{i}\left(\mathcal{B}_{2}\right)} \xi_{(\alpha)}^{i} \partial_{i} V, \alpha=1,2,3
$$

where $\xi_{(\alpha)}^{i}(x)=\beta_{(\alpha) j}^{i} x^{j}$ is a basis for the Lie algebra of $\operatorname{SO}(3), \mathfrak{s o}(3) \cong \mathbb{R}^{3}$, in particular after raising an index we have $\beta_{(\alpha)}^{i j}=\beta_{(\alpha)}^{[i j]}$.

We calculate $\mathbb{T}(0, Q)$ to be

$$
\int_{\mathbf{i}\left(\mathcal{B}_{2}\right)} \beta^{i}{ }_{n} Q^{n}{ }_{j} x^{j} B_{i k} Q^{k}{ }_{m} x^{m}=\beta^{i n} B_{k i} Q^{n}{ }_{j} J^{j m} Q^{k}{ }_{m}
$$

where

$$
Q^{n}{ }_{j} J^{j m} Q_{m}^{k}=\left(Q J Q^{t}\right)^{n k}
$$


expresses the fact that rotating the body by $Q$ induces an orthogonal similarity transformation of the inertia tensor $J$. In particular, there is a $Q_{0}$ such that $J^{0}=$ $Q J Q^{t}$ is diagonal. Due to the fact that $\beta^{\text {in }}$ is skew, we have

$$
\beta^{i n} B_{k i} J_{n k}^{0}=\frac{1}{2} \beta^{[i n]}\left[B, J^{0}\right]_{i n}=0
$$

since by assumption $B_{k i}$ is diagonal. Thus, after applying a rotation to $\mathcal{B}_{2}$, we may without loss of generality assume that $J^{i j}$ is diagonal, $J^{i j}=\rho^{i} \delta^{i j}$ so that we have

$$
\mathbb{T}(0, I)=0
$$

We now calculate $\partial_{Q} \mathbb{T}(0, I) . \mu$ for $\mu \in \mathfrak{s o}(3)$, i.e. $\mu=-\mu^{t}$. We have

$$
\partial_{Q}\left(Q J Q^{t}\right) \cdot \mu=[\mu, J]
$$

Let $\mathfrak{g l}(3)$ be the space of $3 \times 3$ matrices, and for $A \in \mathfrak{g l}(3)$, let $\operatorname{Ad}_{A}$ be the linear operator defined by $\operatorname{Ad}_{A} B=[A, B]$. Recall that the Frobenius inner product on the space of matrices is $\langle A, B\rangle_{\mathcal{F}}=\operatorname{tr} A B^{t}$. We can now write

$$
\partial_{Q} \mathbb{T}(0, I)=-\left|\mathcal{B}_{2}\right|\left\langle\beta, \operatorname{Ad}_{B} \operatorname{Ad}_{J} \mu\right\rangle_{\mathcal{F}}
$$

We observe that $\operatorname{Ad}_{B}$ maps $\mathfrak{s o}(3) \rightarrow \mathfrak{s y m}(3)$ for $B \in \mathfrak{s y m}(3)$. If $B$ is diagonal, which is the case we are considering, then $\operatorname{Ad}_{B}$ maps $\mathfrak{s o}(3)$ into the three dimensional subspace of $\mathfrak{s y m}(3)$ consisting of symmetric matrices with vanishing diagonal elements. Further, $\operatorname{Ad}: \mathfrak{s y m}(3) \rightarrow \mathfrak{s o}(3)$. Thus we have $\operatorname{Ad}_{B} \operatorname{Ad}_{J}: \mathfrak{s o}(3) \rightarrow \mathfrak{s o}(3)$. For $B \in \mathfrak{s y m}(3)$, then using the cyclic property of the trace, we have

$$
\left\langle A, \operatorname{Ad}_{B} C\right\rangle_{\mathcal{F}}=\left\langle\operatorname{Ad}_{B} A, C\right\rangle_{\mathcal{F}},
$$

i.e. $\operatorname{Ad}_{B}$ is self-adjoint with respect to the Frobenius inner product. Hence, $\partial_{Q} \mathbb{T}(0, I)$ is self-adjoint, and

$$
\left\langle\partial_{Q} \mathbb{T}(0, I) \cdot \mu, \beta\right\rangle_{\mathcal{F}}=-\left|\mathcal{B}_{2}\right|\left\langle\operatorname{Ad}_{B} \beta, \operatorname{Ad}_{J} \mu\right\rangle_{\mathcal{F}}
$$

It follows from this identity that if $\operatorname{Ad}_{B}$ and $\operatorname{Ad}_{J}$ have trivial kernel on $\mathfrak{s o}(3)$, then $\partial_{Q} \mathbb{T}(0, I)$ is invertible. Since $J$ is diagonal, $J_{i j}=\rho_{i} \delta_{i j}$, we have

$$
\left(\operatorname{Ad}_{J} \mu\right)_{i j}=\left(\rho_{i}-\rho_{j}\right) \mu_{i j}
$$

and hence

$$
\left\|\operatorname{Ad}_{J} \mu\right\|_{\mathcal{F}} \geq \min _{i \neq j}\left|\rho_{i}-\rho_{j}\right|\|\mu\|_{\mathcal{F}}
$$

It follows that if the Hessian $B_{i j}$ and the inertia tensor $J_{i j}$ of $\mathcal{B}_{2}$ are both diagonal, and such that $B_{i j}$ is invertible with distinct eigenvalues and $J_{i j}$ has distinct eigenvalues, then $\partial_{Q} \mathbb{T}(0, I)$ is invertible. Recalling that the assumption that $B_{i j}$ and $J_{i j}$ are diagonal can be imposed without loss of generality, an application of the implicit function theorem proves the following proposition.

Proposition 4.1. Assume that the Newtonian potential $V_{1}$ of $\mathbf{i}\left(\mathcal{B}_{1}\right)$ has a critical point $\mathcal{O}$ such that the Hessian of $V_{1}$ at $\mathcal{O}$ is invertible and has distinct eigenvalues. Then for any given body $\mathcal{B}_{2}$ such that the inertia tensor $J$, given by (4.2), has distinct eigenvalues, then for small $\lambda>0$, there is a homothetic motion $F x=$ $\lambda(Q x+p)$ such that $F \mathbf{i}\left(\mathcal{B}_{2}\right)$ is equilibrated in the Newtonian potential of $\mathcal{B}_{1}$, i.e. equation (4.3) holds.

Remark 4.1. The existence of reference configurations satisfying the assumptions of proposition 4.1 was shown in [3, section 5.2]. 

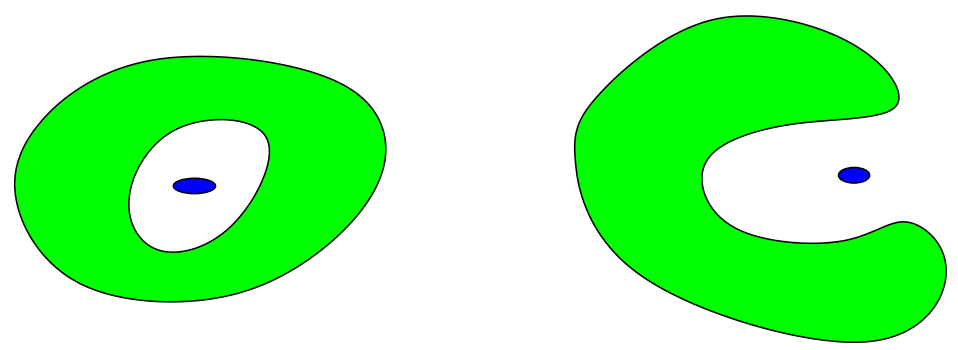

Figure 1. Two-body configurations of the type constructed in Theorem 4.2

We can now apply the results of section 3 , in particular corollary 3.3 to deduce the existence of a class of static, elastic two-body systems. Figure 1 illustrated the type of configurations which are covered by this result.

Theorem 4.2. Assume that the Newtonian potential $V_{1}$ of $\mathbf{i}\left(\mathcal{B}_{1}\right)$ has a critical point $\mathcal{O}$, located in the complement of $\mathbf{i}\left(\mathcal{B}_{1}\right)$, such that the Hessian of $V_{1}$ at $\mathcal{O}$ is invertible and has distinct eigenvalues, and let $\mathcal{B}_{2}$ be a given body such that the inertia tensor $J$ of $\mathcal{B}_{2}$ has distinct eigenvalues.

Then, for sufficiently small $G$, there is a static, self-gravitating elastic two-body system in Einstein gravity close to the configuration $\mathbf{i}\left(\mathcal{B}_{1}\right) \cup F \mathbf{i}\left(B o_{2}\right)$, with $F$ a homothetic motion as constructed in proposition 4.1.

4.2. Axisymmetric bodies. In this section we consider configurations which have a rotational symmetry. We may without loss of generality assume that $\mathbf{i}\left(\mathcal{B}_{1}\right)$ and $\mathbf{i}\left(\mathcal{B}_{2}\right)$ have have centers of mass at the origin $\mathcal{O}$ of the cartesian coordinate system on $\mathbb{R}_{\mathcal{S}}^{3}$, and that the axis of symmetry of the bodies is aligned with the $z$ axis. Thus letting $\phi$ be defined by $\tan \phi=y / x$, we have that $\partial_{\phi}$ is a symmetry of the configuration, in the sense that $\partial_{\phi} \chi_{\mathcal{B}_{\ell}}=0, \ell=1,2$.

Suppose the Newtonian equilibrium condition (2.1) holds. Let $Z=Z(G, A)$ be the solution to the projected system, constructed using Proposition 2.1 and let $\mathbb{N}$ be the normalized force map as in section 3.2 . For the present purpose it is convenient to take $\mathbb{N}$ as defined in terms of the material frame, i.e.

$$
\langle\mathbb{N}, \xi\rangle=G^{-1} \int_{\mathcal{B}_{2}} \xi^{i} b_{i}
$$

As defined, $\mathbb{N}$ takes values in the dual of the space of Killing fields of $\left(\mathbb{R}_{\mathcal{S}}^{3}, \hat{\delta}\right)$. However, using the Euclidean geometry of $\mathbb{R}_{\mathcal{S}}^{3}$, we may consider $\mathbb{N}$ as taking values in the space of Killing fields. We have $\left[\mathbb{N}, \partial_{\phi}\right]=0$. One easily checks that the only Killing fields which have vanishing Lie bracket with $\partial_{\phi}$ are linear combindations of $\partial_{\phi}$ and $\partial_{z}$. Since $\partial_{\phi}$ is a symmetry of the body, it then follows that $\mathbb{N}$ is proportional to $\partial_{z}$. We remark that this can be seen directly from the fact that due to the axisymmetry of the body, any load must be along the $z$-axis.

From the above discussion, it follows that the two components cannot be separated in the $z$-direction. Thus, any axi-symmetric reference configuration with two components must have the property that one component is located "inside" the other, see figure 2, In this figure, each point corresponds to a circle, i.e. the bodies constructed are achieved by rotating the regions shown around the $z$-axis. 

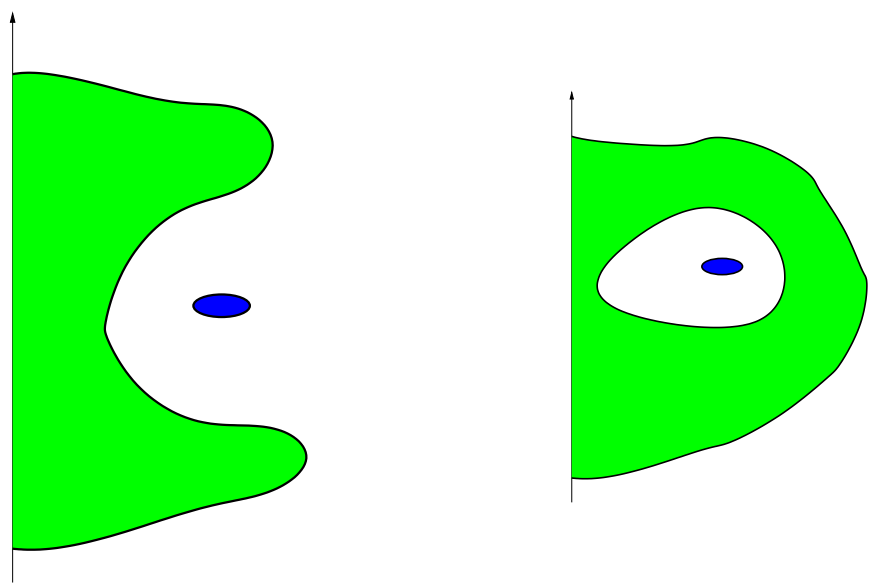

FiguRE 2. Slices through configurations of the type considered in theorem 4.4 Each point in the figure corresponds to a circle.

In each case, a toroidal object is in equilibrium near, or in a toroidal cavity in a larger object.

Thus, in order to solve the equation $\mathbb{N}(G, Z(G, A))=0$, it suffices to consider the $\partial_{z}$ component of $\mathbb{N}$. Further, the only motions we need to consider are those of the form $z \rightarrow z+a$.

The terms in the derivative $\partial_{A} \mathbb{N}(0, I)$ which we need to consider are thus of the form

$$
\int_{\mathbf{i}\left(\mathcal{B}_{2}\right)} \partial_{z}^{2} V_{1}
$$

If this quantity is non-zero, then in view of the remarks above, the argument which proves Proposition 3.1 proves

Proposition 4.3. Assume that the reference body $\mathcal{B} \subset \mathbb{R}_{\mathcal{B}}^{3}$ is in equilibrium in the sense that (2.1a) holds. Let $Z=Z(G, A)$ be the solution to $\mathbb{P}_{\mathcal{B}_{1} \cup \mathcal{B}_{2}} \mathcal{F}=0$ constructed in Proposition 2.1, and let $\mathbb{N}(G, A)$ be the normalized force map defined by (3.3).

Suppose $\mathcal{B}$ is axisymmetric, with its axis of symmetry aligned with the $z$-axis, and with center of mass at the origin $\mathcal{O}$. Further, assume that $\mathcal{B}$ is a disjoint union $\mathcal{B}=\mathcal{B}_{1} \cup \mathcal{B}_{2}$ with the property that

$$
\int_{\mathbf{i}\left(\mathcal{B}_{2}\right)} \partial_{z}^{2} V_{1}
$$

is nonzero, where $V_{1}$ is the potential of $\mathbf{i}\left(\mathcal{B}_{1}\right)$. Then there is an $\epsilon>0$ and a smooth map $G \mapsto A,[0, \epsilon) \rightarrow \mathcal{A}$ with $A(G)$ of the form $z \mapsto z+$ a for $a \in \mathbb{R}$, such that

$$
\mathbb{N}(G, A(G))=0
$$

Remark 4.2. Proposition 4.3 gives an example of a situation where the normalized force map $\mathbb{N}(G, A)$ has degenerate derivative at $(0, I)$, but where the symmetries of the situation still allow us to apply the same argument as in the non-degenerate situation. 


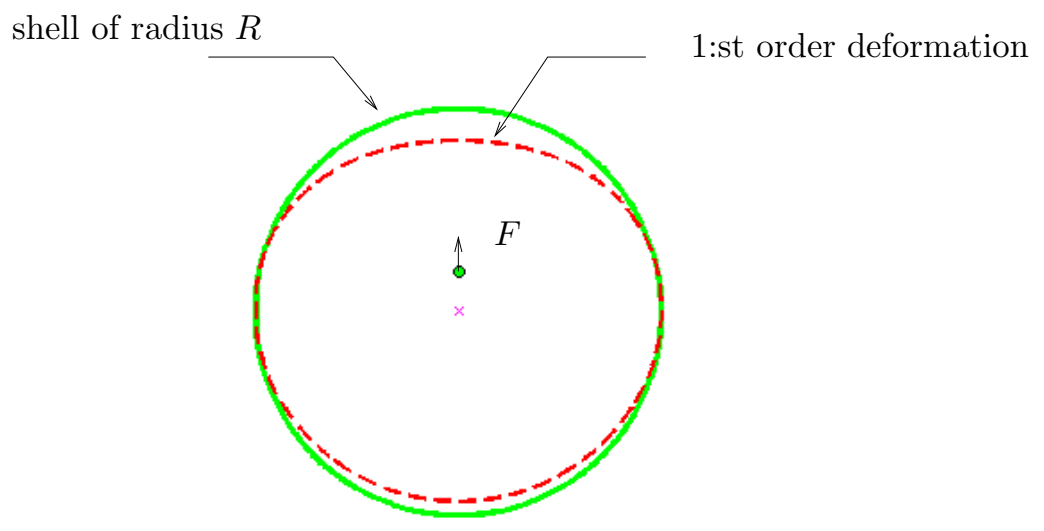

Figure 3. The force generated on a body placed off-center in a spherical, elastic shell.

Theorem 4.4. Suppose that the reference configuration satisfies the assumptions of proposition 4.3. Then, for sufficiently small $G$ there is a static, axisymmetric, self-gravitating two-body system in Einstein gravity, close to the configuration $\mathbf{i}(\mathcal{B})$.

4.3. A body in a spherical shell. As mentioned in the introduction, the Newtonian potential inside a spherical shell consisting of a homogenous material is constant. Considering only rigid bodies in the Newtonian theory, it is thus possible to place a small body at rest at an arbitrary position inside the shell. Here we point out that if we consider instead elastic bodies, then this general construction is no longer possible. On the contrary, we argue here that the only static configuration of this type consists of a spherically symmetric body placed at the center of the shell.

Consider an outer outer shell of radius $R$. Take coordinates such that the center of the ring is at $r=0$. Place the small body at distance $d$ from the center on the $z$-axis and consider the system in Newtonian gravity.

Let us consider linearized elasticity for this system. There linearized deformation of the inner body is zero because the force vanishes in the interior. Due to the principle of actio est reactio, the force of the inner body on the outer shell is equilibrated, and hence the linearized elasticity equation has a solution.

The Newtonian potential generated by the small body is proportional to

$$
\frac{1}{r}+\frac{d z}{r^{3}}+O\left(\frac{1}{r^{3}}\right)
$$

The leading order after the spherical field is a dipole field, i.e. a $\ell=1$ spherical harmonic. Figure 3 shows the upper half of the shell, with the small body. The deformation and the resulting force are indicated in the figure.

The linearized deformation generated by $V$ will therefore generate inside as dominating contribution a $l=1$ spherical harmonic gravitational field

$$
\delta V=a z
$$


However, in this field the small body at $d$ can never be equilibrated (recall that the $l=1$ part is the dominating contribution provided the radius of the outer shell is suffciently large.) Since the linearized problem has no solution, we cannot expect a solution to the full non-linear problem to exist.

Acknowledgements. LA thanks the Mittag-Leffler Institute, Djursholm, Sweden, where part of this paper was written, for hospitality and support.

\section{REFERENCES}

[1] Lars Andersson, Robert Beig, and Bernd G. Schmidt, Static self-gravitating elastic bodies in Einstein gravity, Comm. Pure Appl. Math. 61 (2008), no. 7, 988-1023.

[2] Robert Beig and Bernd G. Schmidt, Relativistic elastostatics. I. Bodies in rigid rotation, Classical Quantum Gravity 22 (2005), no. 11, 2249-2268.

[3] _ Celestial mechanics of elastic bodies, Math. Z. 258 (2008), no. 2, 381-394.

[4] Robert Beig and Richard M. Schoen, On Static n-body Configurations in Relativity, Class. Quant. Grav. 26 (2009), 075014.

[5] S. Chandrasekhar, Ellipsoidal figures of equilibrium, New York : Dover, 1987., 1987.

[6] Herbert Goldstein, Classical mechanics, second ed., Addison-Wesley Publishing Co., Reading, Mass., 1980, Addison-Wesley Series in Physics.

[7] Müller zum Hagen, PhD thesis.

E-mail address: larsa@math.miami.edu

Albert Einstein Institute, Am Mühlenberg 1, D-14476 Potsdam, Germany and Department of Mathematics, University of Miami, Coral Gables, FL 33124, USA

E-mail address: bernd@aei.mpg.de

Max-Planck-Institut für Gravitationsphysik, Albert-Einstein-Institut, Am Mühlenberg 1, D-14476 Golm, Germany 